\title{
Evidence For The Involvement of B Cells and Antibody in The Pathogenesis of Multiple Sclerosis in Immunized Mouse With Recombinant Myelin Basic Protein Peptide
}

Maryam Sahlolbei ( $\triangle$ marysahl@gmail.com )

IUMS: Iran University of Medical Sciences https://orcid.org/0000-0001-8575-1273

Maryam Hajizadeh

IUMS: Iran University of Medical Sciences

Marzie Naseri

IUMS

Hossein Ghanbarian

Shahid Beheshti University

Seyed Mahmoud Hashemi

Shahid Beheshti University

Zahra Madjd

IUMS: Iran University of Medical Sciences

Jafar Kiani

IUMS: Iran University of Medical Sciences

\section{Research Article}

Keywords: Multiple Sclerosis (MS), Antibodies, Humoral Immunity, Recombinant Myelin Basic Protein (rMBP), Immunization

Posted Date: February 23rd, 2021

DOI: https://doi.org/10.21203/rs.3.rs-214919/v1

License: (c) (i) This work is licensed under a Creative Commons Attribution 4.0 International License. Read Full License 


\section{Abstract}

Over the years, regarding great progresses in knowledge of immunology and neuroscience, the treatment of multiple sclerosis (MS) has been changed. The earlier strategies were focused mainly on T lymphocytes as pioneer cells responsible to inflammatory damage in the central nervous system lesions, whereas B cells, plasma cells and antibodies are also found in the active nerve lesions in MS patients. Despite the accumulating evidence, the role of Myelin basic protein (MBP) antibodies in progression of lesions in nervous system is not completely clear yet. In this regard, here, we present data on B cells and antibody level after MBP immunization of MS mice model.

Recombinant fusion protein harboring Myelin basic protein peptide (amino acids 83-99) and CFP was produced in $E$. coli and purified with chromatography. Then, the C57BL / 6 mice were immunized by rMBP-CFP. Antibody-based assay was used to quantify the level of reactivity to the MBP in mice serum. Subsequently, humoral immunity was analyzed by immunohistochemistry (IHC), ELISA, and Flow cytometry. Our data indicated an increase in autoreactive B cells and MBP specific antibodies after immunization. IHC analysis revealed an increasing penetration rate of immune cells and the nerve lesions development in the nervous system following increasing in MBP antibody titers.

This study represented data to support this idea that reactive B cells and antibodies to MBP may contribute to MS pathogenesis. Hence, targeting of these autoreactive B cells and antibodies can be used as potential tools in treatment of MS patients.

\section{Introduction}

Multiple sclerosis (MS) is a chronic autoimmune inflammatory disease that characterized by two major pathological hallmarks in the central nervous system (CNS), the plaques or lesions composed by multiple focal areas of myelin loss as well inflammation damage within the CNS. These neurotic cells damage leads to a cascade of immune cells interactions and antibody activation resulted in a neurological disability in young adults (Cepok et al., 2005; Lee, Gauna, Pauley, Park, \& Cha, 2012; Pandit \& Murthy, 2011). Although MS-related immune responses initiate as T-cell-mediated response, according to clinical trials data, B cells and antibodies play a pivotal role in MS as well as other autoimmune diseases (Owens, Bennett, Gilden, \& Burgoon, 2006).

Three main subgroups of MS, including relapsing-remitting MS (RRMS), secondary progressive MS (SPMS) and primary progressive MS (PPMS), show different histopathological models. The progression of MS accurses in three steps; first a pre-clinical stage, second a relapsing-remitting (RRMS) clinicalstage, which known with discrete features of neurologic dysfunction such as optic neuritis, sensory disturbances or disturbances in motor and cerebellar function; and a progressive clinical-stage during which neurologic dysfunction progressively worsens, affecting, in specific, a patient's walk (Lucchinetti et al., 2008; Wolswijk, 1998). Humoral immunity is thought to have an important effect on the inflammation and development of demyelination lesions. Antibody activation against neurotic cells is a sign of MS that 
founded in the brain parenchyma, meninges and cerebrospinal fluid (CSF) of patients (Baecher-Allan, Kaskow, \& Weiner, 2018; Magliozzi et al., 2007). Increased antibody levels in the spinal cord of MS patients were first reported in the year 1942 by Kabat et al, which revealed the self-reactive antibodies are involved in the pathogenesis that act as an indicator in diagnosis of disease (Kabat, Moore, \& Landow, 1942). Since then, an excess of studies has been performed on the function of antibodies in autoimmune diseases (Weber, Hemmer, \& Cepok, 2011).

Researchers have found the significant correlation between IgG levels in CSF and the progression of MS disease (Genain, Cannella, Hauser, \& Raine, 1999; Ota et al., 1990; Villar et al., 2002). In the acute phase, these autoantibodies accumulate alongside the axons and specifically bind to myelin protein. It seems that antibodies can induce axonal injury, although, the exact antigenic targets have not yet been identified (Derfuss \& Meinl, 2012).

The mice models analysis, from experimental autoimmune encephalomyelitis (EAE) as well-accepted model of MS showed that the injection with the various purified components of the myelin sheath (MBP), proteolipid protein (PLP), and myelin oligodendrocyte glycoprotein (MOG), can induce pathogenesis in EAE model (Janeway Jr, Travers, Walport, \& Shlomchik, 2001). Recently, Berger et al., have reported a promising predictor of clinically definite MS in a cohort, patients who were seropositive for both anti-MOG and anti-MBP had more repeated and earlier relapses than those who were seronegative. The Existence of both antibodies predicted the conversion to definite MS more than the presence of anti-MOG IgM alone (Reindl et al., 1999). MBP comprises approximately $30-40 \%$ of the total proteins in the myelin (Warren \& Catz, 1993). The high level of anti-MBP are usually detected in patients with acute MS and reduced in the chronic stage, it presumes that it has the effect on progression of relapsing-remitting phase (Allegretta, Nicklas, Sriram, \& Albertini, 1990). Although anti-MBP-IgG has seen in early phase, it is probably that the autoantigens can induce disease (DeLuca et al., 2007; Sadovnick, Dyment, \& Ebers, 1997).

Hence, the autoantibodies seem to have an important role in the progression of MS, therefore, it could be an interesting drug target. Autoantibodies recognize myelin and recruit inflammatory cells to focal areas, thereby target the central nervous system myelin sheet (Rozenblum, Kaufman, \& Vitullo, 2014). It's still under debate whether these occurring antibodies indeed actively contribute to the pathogenesis or progression of the disease. Moreover, $\mathrm{T}$ cells and B cells as the most prominent cell types in spleen show a transient increase during the acute phase of MS, whereas, their population are decreased after the acute phase. In addition, the rate of CD4 + and CD8 + T cells are changed in the spleen, during the different stage of EAE disease (Barthelmes et al., 2016). Regarding the increased clinical evidence for the autoantigen presentation during MS progression, we aimed to further explore the MBP-specific antibodies roles. Here, we demonstrated that $B$ cells and MBP antibody have a more significant role in the initiation and progression of the lesions in mice CNS.

\section{Materials And Methods \\ 2.1. Animals and ethics}


All experiments were performed in accordance with the instructions and approval of the animal Research and Ethics Committee of Iran University of Medical Sciences (No: IR.IUMS.REC 1395.9323513001). Four groups of female C57BL/6 mice ( $\mathrm{n}=16,6-8$ weeks old, and with weight range of $18-20 \mathrm{gr}$ ) were obtained from Pasteur Institute of Iran (Tehran, Iran). Animals were housed in a room under standard conditions ( $12 \mathrm{~h}$ light-dark cycle; the temperature of $23 \pm 1^{\circ} \mathrm{C}$ ). Four animals were maintained in each cage (polypropylene cages; $42 \times 27 \times 15 \mathrm{~cm}$ ), and food and water were provided. Mice were selected at random for each experimental and control groups. The experiments were carried out as blind to prevent any bias in the study results.

\subsection{Vector construction}

pET28a/ MBP-CFP plasmid (kanamycin-resistant; Novagen, USA) containing T7 promoter was used to express recombinant rMBP-CFP Recombinant plasmids were chemically transformed into the $B L 21$ strain of $E$. coli (DE3) by $\mathrm{CaCl} 2$ (Chan, Verma, Lane, \& Gan, 2013). Plasmids from an overnight culture were then isolated using the purification kit according to the manufacturer's protocol (Qiagen, USA).

\subsection{Protein expression and purification}

BL21 (DE3) containing rMBP-CFP were inoculated into $500 \mathrm{~mL}$ LB medium supplemented with antibiotics

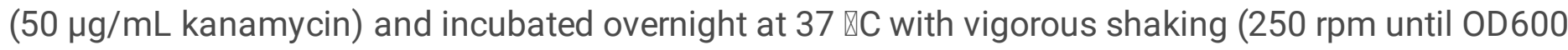
reached). Then, the cells were induced with isopropyl $\beta$-D-1-thiogalactopyranoside (IPTG) to the final concentration of $0.5 \mathrm{mM}$ and incubation was continued for $5 \mathrm{~h}$ at $37 \otimes \mathrm{C}$ with shaking. The cells were then harvested by centrifugation (4000 rpm / $10 \mathrm{~min}$ ) and the wet pellet was weighed and stored in a $-20^{\circ} \mathrm{C}$ freezer until further purification Then frozen bacterial pellets were broken in lysis buffer (Lysozyme $10 \mu \mathrm{g} / \mathrm{ml}$, PMCF $1 \mu \mathrm{g} / \mathrm{ml}$ ) by three cycles of freeze and thaw. The lysate was centrifuged (12,000 rpm, $4{ }^{\circ} \mathrm{C}$, for $20 \mathrm{~min}$ ) and the supernatant was removed. The protein was isolated using a Nickel column (NiNTA kit) according to the manufacturer's protocol. The protein extract was detected by UV280, confirmed by $12 \%$ SDS-PAGE and then was concentrated by Amicon ${ }^{\circledR}$ Ultra Centrifugal Filter and quantitated by the Bradford method.

\subsection{Immunization and challenge}

Immunization was induced in $C 57 B L / 6$ mice by rMBP-CFP (83-92). Eight female mice were immunized with an encephalitogenic cocktail containing rMBP-CFP (50 mg/mouse) and complete Freund's adjuvant (Sigma Co, USA). The $100 \mu \mathrm{L} /$ mice emulsion was injected subcutaneously (s.c) and was waited 2 weeks to build up a primary immunological response. For second and third boosting doses, $30 \mathrm{mg} / \mathrm{mice}$ of rMBP-CFP was added to Freund's Incomplete Adjuvant (FIA). The C57BL/6 mice ( $n=16)$ were divided into three test groups ( $n=4$ per group) and one control group $(n=4)$. To investigate the humoral immunity and its effect on CNS demyelination, the 42-days trial was proceeded according to the scheme summarized in Fig. 1. After immunization, the clinical evaluation of EAE mice ( 0 , healthy; 0.5 , flaccidity and partial paralysis of the tail; 1 , complete paralysis of the tail; 1.5 , weakness in one hind limb; 2 , weakness in both hind limb; 2.5, partial hind limb paralysis; 3 , complete hind limb paralysis; 3.5 , partial forelimb paralysis; 4 , 
complete forelimb paralysis; 5 , mortality; 6 )and body weight measured according to the standard protocol(Racke, 2001) and the process was blindly registered.

\subsection{SDS-PAGE and Western blotting}

The purified rMBP-CFP protein was analyzed by sodium dodecyl sulfate-polyacrylamide gel electrophoresis (12\% SDS-PAGE). To check the antibodies rMBP-CFP, the serum was isolated from the mice blood before and after immunization and was analyzed using western blot. First, $1 \mu \mathrm{g}$ of protein was loaded onto $12.5 \%$ SDS-PAGE, and was transferred to a polyvinylidene difluoride (PVDF) transfer membrane using the electrophoresis apparatus (Bio-Rad, Mini Trans-Blot). Briefly, the membrane was incubated in blocking buffer ( $5 \%$ non-fat milk) for 30 minutes, then, the membrane was washed in a wash buffer and incubated with primary antibody (mouse serum1:100 dilution) for overnight. After that was incubated with secondary antibody (Goat anti-mouse $\lg$ G 1:1000 dilution), and was washed three times. Finally, the color was developed by incubating the membrane in alkaline phosphatase buffer containing chemiluminescence detection with ECL (Promega).

\subsection{Tissue preparation and Histological and Immunohistochemistry examination}

Mice were sacrificed, spinal cords were separated and perfused with $25 \mathrm{~mL}$ cold PBS, then were fixed in $4 \%$ paraformaldehyde for overnight. Tissues were paraffin-embedded and sectioned at $5 \mu \mathrm{m}$ by the Developmental Biology Histology core at Firozgar Hospital. Representative sections were stained with Luxol Fast Blue stain (LFB) to detect myelin detrition, and Hematoxylin and Eosin staining (H \&E) were carried out to detect inflammation according to the standard protocol (Feldman \& Wolfe, 2014). Slides were examined by light microscopy using a Nikon 90i motorized upright digital microscope and analyzed by image $\mathrm{J}$ software. Fluorescent staining was applied to observe T cells (CD3) and autoreactive B cells (CD-45R) infiltration in the spinal cord and stained samples were analyzed microscopically under fluorescent/FITC filter and image $\mathrm{J}$ software. All data were analyzed by Prism software. Histological analysis was repeated three times for each independent experiment.

\subsection{ELISA analysis}

Blood samples were collected from mice before immunization and on days 14, 28, and 42 and were allowed to clot at room temperature. Serum was obtained by centrifugation of clotted blood at 15,000×g for $30 \mathrm{~min}$ and were frozen at $-20 \circ \mathrm{C}$ until use. Total serum MBP-specific IgG was quantified by plating serial serum dilutions on 96-well plates pre-coated with rMBP-CFP (with concentration of $5 \mathrm{mg} / \mathrm{ml}$ ) in the coating buffer (sodium bicarbonate). Plates were blocked ( $5 \%$ nonfat dry milk containing $0.05 \%$ PBST) and incubated with sera overnight at $4{ }^{\circ} \mathrm{C}$. After washing, Goat anti-mice IgG- HRP (1:250) were detected with alkaline phosphatase-conjugated goat anti-mouse lgG. The enzymatic reaction were stopped by adding $50 \mu$ of $2 \mathrm{~N}$ sulfuric acid and optical density were measured at $450 \mathrm{~nm}$ using an ELISA reader.

\subsection{Isolation of PBMC from mouse spleen and Flow cytometry analysis}


The spleens were harvested from immunized and control mice and Peripheral Blood Mononuclear Cells (PBMC) were isolated by lysis buffer. After centrifugation, cells were incubated with antibodies (concentrated to $1 \times 10^{6}$ cells $/ \mathrm{ml}$ ). The following antibodies were used for two-color flow cytometric analysis: FITC-Anti-Mouse CD45R, PE- Anti-Mouse CD19 (Bio Legend) product for detecting autoreactive B cell, and PE-Anti-Mouse CD4 and APC-Anti-Mouse CD8 (Bio Legend) to show T lymphocytes. Cells were acquired on a BD flow cytometer and analyzed with Flow Jo software. The rMBP-CFP protein was used for detection B Cells, which release anti MBP antibody. PBMC samples collected after immunization by rMBP-CFP from each mice. PBMC (concentration to $1 \times 10^{6}$ cells $/ \mathrm{ml}$ ) were mixed with $50 \mu \mathrm{M}$ of rMBPCFP $(1 \mathrm{mg} / \mathrm{ml})$ and incubated for $1 \mathrm{~h}$ in dark then washed, for detecting autoreactive B cell. Cells were acquired on a BD flow cytometer and analyzed with Flow Jo software.

\subsection{Statistical Analysis}

The two-way ANOVA comparisons test was used for comparisons of clinical score severity in mice. Tukey's Multiple Comparison test was used for comparisons between ELISA and immunohistochemistry analyses by the statistical package of Prism 3.03 (Graph Pad, USA). The P-value of less than 0.05 was considered statistically significant.

\section{Result}

\subsection{Characterization of rMBP-CFP}

The expression of rMBP-CFP was induced by IPTG, as described. The cells were harvested, lysed and processed for recombinant proteins purification in two steps, first on a Ni column and then by Amicon ${ }^{\circledR}$ Ultra filters. The purity of the protein product was determined by SDS-PAGE.

\subsection{Neurological function and clinical score induced by rMBP-CFP immunization}

To investigate the clinical disease presentation, the C57BL/6 mice were immunized using rMBP-CFP in three steps. The neurological effects of immunization by rMBP-CFP on test group were evaluated daily until 21 days after symptoms onset and were compared to control group (PBS). The onset of clinical symptoms occurred between 11 to14 days after immunization. The day of disease manifestation and the severity of its symptoms in test group were compared to the control group. The mean clinical score of PBS- and rMBP-CFP -treated mice were $0 \pm 0$ and $2.10 \pm 0.22$, respectively. The score were increased in rMBP-CFP treated mice during the follow up (Fig. 2A). The average weight increasing in the PBS- and rMBP-CFP -treated mice were $3.43 \pm 0.22$ - and $1.16 \pm 0.30$ grams, respectively (Fig. 2B). Statistical comparison with the two-way ANOVA method indicates that there is a significant difference in weight between the immunized group and control group, also this analysis for clinical score is significate too. ( $P$ $<0.0001)$.A comparison of the score and weight of the two groups confirmed that the rMBP-CFP can induce the clinical symptoms in immunized mice. 


\section{3 rMBP-CFP immunization and effect on humoral immune response in mice}

Two experiments were performed to determine the humoral immune response to rMBP-CFP peptide. Initially, using western blotting, the rMBP-CFP peptide was probed with a pool of sera obtained from each group of immunized mice. Strong antibody responses were induced by immunization against rMBP-CFP in three test groups as shown in Fig. 3A. There was no antibody response in control group (PBS). In the second set of experiments, the IgG antibody kinetics and frequency were analyzed in sera obtained from the mice prior to immunization and 14, 28, and 42 days after immunization using ELISA. As shown in Fig. 3B, the IgG antibodies in sera obtained from immunized mice with rMBP-CFP protein show an increase at days 14, 24, and 42 compared to before immunization. Tukey's Multiple Comparison Test elicited, the statistically significant between day 42 and day prior to immunization $(P=0.0346)$.

\section{4. rMBP-CFP improved neuropathology in immunization mice}

The spinal cord is the early site of inflammatory damage in murine system of EAE (Ingunn M Stromnes \& Joan M Goverman, 2006). Hence, mice spinal cords were examined for pathologic changes. Luxol Fast Blue staining exhibited demyelinated areas in the lumbar spinal cord of rMBP-CFP immunized mice (Fig. 4A). Tukey's Multiple Comparison Test analysis showed a significant difference in mice two weeks after the first injection compared to 6 weeks after the first injection. Furthermore, in line to increasing the level of antibody, development of demyelinated area was observed (Fig. 4B). In order to evaluate the inflammation, H\&E staining from the spinal cord of animals were performed. Inflammatory infiltrates mainly localized around the central canal and marginal zone in rMBP-CFP immunized mice (overlapping the demyelination areas). The localization and size of the inflammation were different in three test groups, although no significant difference were observed between groups (Fig. 4C).

\subsection{Cell Infiltration in the CNS}

To study the lymphocyte cells' infiltration in CNS, we used fluorescent staining of immune cell markers. Lymphocytes were observed in the periphery area and were present in the inflammatory infiltrates. Concurrently by increased antibody, the T cell (CD3) and autoreactive B cell (CD-45R) infiltration were also observed mainly in demyelination areas (Fig. 5A and B). Tukey's Multiple Comparison Test analysis showed a significant difference in autoreactive B cell (CD-45R) and T cell (CD3) infiltration by the expression level of CD-45R and CD3 markers in the first group (two weeks after the first injection) compared to the third group (6 weeks after the first injection) (Fig. 5C and D).

\subsection{Cell mediate immune response to rMBP-CFP in immunized mice}

Due to the limitation of CSF in flow cytometry analysis including low cellularity and rapid declining of WBC viability, we performed flow cytometry on PBMCs from mice spleen. Evaluation of CD $4+$ and CD $8+$ 
T cells levels in spleen samples, showed no significant differences in T CD8 + cells in immunized mice compared to control (both after 42 days). But T CD $4+$ cells in spleen of rMBP-CFP immunized mice showed a significant decrease compared to control group $(P<0.0001)$ (Fig. 6A)

Next, the presence of CD19 + cells in spleens of rMBP-CFP immunized mice was assessed. Our results showed that the B cell population represents $\sim 30-35 \%$ of total spleen cells in non-immune mice, whereas after the rMBP-CFP immunization, the percentages of CD19+ cells was increased. Splenocytes from C57BL/ 6 mice were stained using the CD45R (also known as B220) to identify active B cells from conventional B cells (CD19+), both B cells express high levels of the CD19 molecule, but active B cells express higher levels of CD45R. The population of $B$ cells in the immunized group shifted to active $B$ cells indicated by increased active $B$ cell (CD19+/CD45R+) populations in mice spleen after rMBP immunization (Fig. 6B). Immunization of mice with rMBP increased active B cell (CD19 + / CD45R +) cells in the mouse spleen, but after the acute phase, the active $B$ cell reduced. To ensure the specifically activation of $B$ cells against $r M B P$, PBMCs from the immunized mouse were incubated with rMBP-CFP and then flow cytometry were performed to detect the CFP positive cells as $B$ cells population that specifically have produced antibody against rMBP. The results showed that $7.9 \%$ of $B$ cells were CFP positive cells (Fig. 6C).

\section{Discussion And Conclusions}

During the last years, our knowledge regarding the role of immune cells and antibodies in the autoimmune disorders has been progressed tremendously (Bar-Or et al., 2010; Li, Patterson, \& Bar-Or, 2018). The emerging evidences about $B$ cells and their pathological roles in autoimmune diseases have conducted to B-cell depletion therapy, not only in diseases which are clearly B cell dominated but also in autoimmune diseases that are traditionally viewed as T cell-mediated including MS (Hampe, 2012). In autoimmune disease, breakdown the self-tolerance to self-antigens or modified self-antigens can lead to the generation of autoantibodies. After the starting of autoantibody production, inflammation causes the release of intracellular antigens, leads to activation of antibody, and stimulation of clinical symptoms resulted in the extension of the autoreactive B cell clones (Suurmond \& Diamond, 2015).

Based on accumulating evidences, the pathophysiology of Multiple sclerosis has principally mediated by helper $T$ cells which provided insights to developing promising treatment strategies. This treatment decreases the relapse rate by approximately one third but does not fully prevent the occurrence of exacerbations (Hauser et al., 2008). Other studies showed that autoimmune B cells and humoral immune mechanisms also play the key roles in disease progression (Owens et al., 2006). Preclinical models even specifically targeted autoantibody-specific B cells and plasma cells, which can be considered as a treatment, also increased the evidence of involvement of B lymphocytes in MS (Ludwig et al., 2017). Recently, molecular analyses showed that passive transfer of immunogens can lead to inflammation in the nerves, although all antibodies do not have equal capacity to do so (Mannara et al., 2012; Ingunn M. Stromnes \& Joan M. Goverman, 2006). B cells, plasma cells and antibodies are commonly found in active nerves lesions and have a role in the formation, maintenance, and development of new lymphoid 
foci in the CNS, although little is still known about B cell response in the MS (McMillan, Longmire, \& Yelenosky, 1976; Stork et al., 2018; Weber et al., 2011).

Despite recent advances in long-term immunomodulatory treatment of MS, first-line option to treat the MS relapses mainly relies on Glucocorticosteroids (GCS) and second-line is commonly therapeutic plasma exchange (TPE). TPE is a strategy to reduce the level of antibodies in the serum in severing attacks (Rolfes et al., 2019; Stork et al., 2018). To develop the safe treatment option for MS patients, several therapeutic monoclonal antibodies and agents were created including; CD20-depleting antibodies, BAFF/APRIL-depleting agents, Alemtuzumab, Natalizumab, FTY720, IFN- $\beta$, Glatiramer acetate, steroids, and plasma exchange on B-cell immunology (Pröbstel, Sanderson, \& Derfuss, 2015; Voge \& Alvarez, 2019). Targeting the antigen-specific immune response and antigen-Specific $B$ cell depletion, are promising strategies (Musette \& Bouaziz, 2018). The positive result of B cell targeting therapies emphasizes the main roles of B cells in the pathogenesis of autoimmune diseases (Hofmann, Clauder, \& Manz, 2018). Therefore, targeting the humoral immune system and B cell depletion would be a reasonable therapeutic option in MS.

Most Multiple sclerosis research is about protein myelin oligodendrocyte glycoprotein rather than MBP or PLP. However, increasing evidence confirmed that the autoimmune reactivity against other CNS-specific myelin proteins could involve in the pathogenesis of MS (Ben-Nun et al., 1996). Importantly, the MBP epitope was recognized by whit conjunction with four different HLA-DR molecules, in spanning amino acids 87-106 region (R Martin et al., 1991). Data obtained from the brain analysis of demyelinating mouse model confirmed the view that the changes in the human brain are probably related to the MBP pathogenesis. Hence, the anti-MBP antibody is a candidate for autoimmune processing (Roland Martin et al., 1990). These findings can have important implications in designing of therapeutic strategies for MS.

We believe that our study may provide new insight in MS pathogenesis; however, the targets of these antibodies remain unclear. Our findings from immunization using purified rMBP-CFP recombinant protein in complete Freund adjuvant revealed the strong immune responses in immunized mice as shown by a significant increment in IgG titration and induction of demyelination in CNS. Histopathological comparison in accompanied to the humoral immune response showed increased immune cell infiltration and developing of inflammatory demyelinating lesions in CNS. In conclusion, this study confirms association between MBP autoantibody and demyelination in MS, in other word the involvement of MBPspecific antibodies in MS pathogenesis and progression. In addition to diagnostic application of these antibodies in MS, they also can use to designing of therapeutic strategies. Reducing the MBP autoantibody levels through antibody removal from patient sera and targeting of autoreactive $B$ cells maybe be effective and promising tools in reducing nerve tissue damage and improve the disease symptoms. However, further investigation is needed to verify this hypothesis. It is may be able to prevent the progression of myelin damage in RRMS. Further understanding of B cell function and secretion of antibodies against nerve cell membranes is useful to find efficient treatment strategy to target the autoreactive B cell. 


\section{Abbreviations}

B6:C57BL/6 CFA: Complete Freund's adjuvant

CNS: Central nerves system EAE: Experimental autoimmune encephalomyelitis

H \&E: Hematoxylin and eosin stain LFB: Luxol fast blue stain

MOG: Myelin oligodendrocyte glycoprotein MS: Multiple sclerosis

rMBP: Recombinant myelin basic protein RRMS: Relapsing-remitting Multiple sclerosis

\section{Declarations}

\section{- Acknowledgement}

This project was supported financially by research grant No: 96-04-193-32722 Iran University of Medical Sciences, Tehran, Iran and the Research Department of Shahid Beheshti University of Medical Sciences (Grant No. 12246).

\section{- Conflict of interest statement}

The authors declare that the research was conducted in the absence of any commercial or financial relationships that could be construed as a potential conflict of interest.

\section{- Ethics approval}

This study and handling of mice, in general, was conducted in strict accordance with the principles outlined in the Iran University of Medical Sciences, Tehran, Iran animal Protection Law (Number: IR.IUMS.REC 1395.9323513001).

\section{- Funding}

This work was supported by Iran University of Medical Sciences, Tehran, Iran. (Grants No: 96-04-19332722) and the Research Department of Shahid Beheshti University of Medical Sciences (Grant No. 12246).

\section{References}

1. Allegretta, M., Nicklas, J. A., Sriram, S., \& Albertini, R. J. (1990). T cells responsive to myelin basic protein in patients with multiple sclerosis. Science, 247(4943), 718-721.

2. Baecher-Allan, C., Kaskow, B. J., \& Weiner, H. L. (2018). Multiple sclerosis: mechanisms and immunotherapy. Neuron, 97(4), 742-768. 
3. Bar-Or, A., Fawaz, L., Fan, B., Darlington, P. J., Rieger, A., Ghorayeb, C., . . Zhang, J. (2010). Abnormal B-cell cytokine responses a trigger of T-cell-mediated disease in MS? Annals of neurology, 67(4), 452-461.

4. Barthelmes, J., Tafferner, N., Kurz, J., de Bruin, N., Parnham, M. J., Geisslinger, G., \& Schiffmann, S. (2016). Induction of Experimental Autoimmune Encephalomyelitis in Mice and Evaluation of the Disease-dependent Distribution of Immune Cells in Various Tissues. Journal of visualized experiments : JoVE(111), 53933. doi:10.3791/53933

5. Ben-Nun, A., Mendel, I., Bakimer, R., Fridkis-Hareli, M., Teitelbaum, D., Arnon, R., . . Kerlero de Rosbo, N. (1996). The autoimmune reactivity to myelin oligodendrocyte glycoprotein (MOG) in multiple sclerosis is potentially pathogenic: effect of copolymer 1 on MOG-induced disease. J Neurol, 243(4 Suppl 1), S14-22. doi:10.1007/bf00873697

6. Cepok, S., Rosche, B., Grummel, V., Vogel, F., Zhou, D., Sayn, J., . . Hemmer, B. (2005). Short-lived plasma blasts are the main B cell effector subset during the course of multiple sclerosis. Brain : $a$ journal of neurology, 128(7), 1667-1676.

7. Chan, W.-T., Verma, C. S., Lane, D. P., \& Gan, S. K.-E. (2013). A comparison and optimization of methods and factors affecting the transformation of Escherichia coli. Bioscience reports, 33(6), e00086.

8. DeLuca, G. C., Ramagopalan, S. V., Herrera, B. M., Dyment, D. A., Lincoln, M. R., Montpetit, A., ... Sadovnick, A. D. (2007). An extremes of outcome strategy provides evidence that multiple sclerosis severity is determined by alleles at the HLA-DRB1 locus. Proceedings of the National Academy of Sciences, 104(52), 20896-20901.

9. Derfuss, T., \& Meinl, E. (2012). Identifying autoantigens in demyelinating diseases: valuable clues to diagnosis and treatment? Current opinion in neurology, 25(3), 231-238.

10. Feldman, A. T., \& Wolfe, D. (2014). Tissue processing and hematoxylin and eosin staining. In Histopathology (pp. 31-43): Springer.

11. Genain, C. P., Cannella, B., Hauser, S. L., \& Raine, C. S. (1999). Identification of autoantibodies associated with myelin damage in multiple sclerosis. Nature medicine, 5(2), 170-175.

12. Hampe, C. S. (2012). B Cells in Autoimmune Diseases. Scientifica, 2012, 215308. doi:10.6064/2012/215308

13. Hauser, S. L., Waubant, E., Arnold, D. L., Vollmer, T., Antel, J., Fox, R. J., . . Smith, C. H. (2008). B-Cell Depletion with Rituximab in Relapsing-Remitting Multiple Sclerosis. New England Journal of Medicine, 358(7), 676-688. doi:10.1056/NEJMoa0706383

14. Hofmann, K., Clauder, A.-K., \& Manz, R. A. (2018). Targeting B Cells and Plasma Cells in Autoimmune Diseases. Frontiers in Immunology, 9(835). doi:10.3389/fimmu.2018.00835

15. Janeway Jr, C. A., Travers, P., Walport, M., \& Shlomchik, M. J. (2001). The complement system and innate immunity. In Immunobiology: The Immune System in Health and Disease. 5th edition: Garland Science. 
16. Kabat, E. A., Moore, D. H., \& Landow, H. (1942). An electrophoretic study of the protein components in cerebrospinal fluid and their relationship to the serum proteins. The Journal of clinical investigation, 21(5), 571-577.

17. Lee, B. H., Gauna, A. E., Pauley, K. M., Park, Y.-J., \& Cha, S. (2012). Animal models in autoimmune diseases: lessons learned from mouse models for Sjögren's syndrome. Clinical reviews in allergy \& immunology, 42(1), 35-44.

18. Li, R., Patterson, K. R., \& Bar-Or, A. (2018). Reassessing B cell contributions in multiple sclerosis. Nature immunology, 19(7), 696-707.

19. Lucchinetti, C. F., Gavrilova, R. H., Metz, I., Parisi, J. E., Scheithauer, B. W., Weigand, S., . . Brück, W. (2008). Clinical and radiographic spectrum of pathologically confirmed tumefactive multiple sclerosis. Brain : a journal of neurology, 131(Pt 7), 1759-1775. doi:10.1093/brain/awn098

20. Ludwig, R. J., Vanhoorelbeke, K., Leypoldt, F., Kaya, Z., Bieber, K., McLachlan, S. M., . . Hammers, C. M. (2017). Mechanisms of autoantibody-induced pathology. Frontiers in Immunology, 8, 603.

21. Magliozzi, R., Howell, O., Vora, A., Serafini, B., Nicholas, R., Puopolo, M., . . Aloisi, F. (2007). Meningeal B-cell follicles in secondary progressive multiple sclerosis associate with early onset of disease and severe cortical pathology. Brain : a journal of neurology, 130(4), 1089-1104. doi:10.1093/brain/awm038

22. Mannara, F., Valente, T., Saura, J., Graus, F., Saiz, A., \& Moreno, B. (2012). Passive experimental autoimmune encephalomyelitis in C57BL/ 6 with MOG: Evidence of involvement of B cells. PLoS One, $7(12)$, e52361.

23. Martin, R., Howell, M. D., Jaraquemada, D., Flerlage, M., Richert, J., Brostoff, S., . . McFarland, H. F. (1991). A myelin basic protein peptide is recognized by cytotoxic T cells in the context of four HLADR types associated with multiple sclerosis. Journal of Experimental Medicine, 173(1), 19-24. doi:10.1084/jem.173.1.19

24. Martin, R., Jaraquemada, D., Flerlage, M., Richert, J., Whitaker, J., Long, E. O., . . McFarland, H. F. (1990). Fine specificity and HLA restriction of myelin basic protein-specific cytotoxic T cell lines from multiple sclerosis patients and healthy individuals. The Journal of Immunology, 145(2), 540-548.

25. McMillan, R., Longmire, R., \& Yelenosky, R. (1976). The effect of corticosteroids on human IgG synthesis. The Journal of Immunology, 116(6), 1592-1595.

26. Musette, P., \& Bouaziz, J. D. (2018). B Cell Modulation Strategies in Autoimmune Diseases: New Concepts. Frontiers in Immunology, 9(622). doi:10.3389/fimmu.2018.00622

27. Ota, K., Matsui, M., Milford, E. L., Mackin, G. A., Weiner, H. L., \& Hafler, D. A. (1990). T-cell recognition of an immuno-dominant myelin basic protein epitope in multiple sclerosis. Nature, 346(6280), 183187.

28. Owens, G. P., Bennett, J. L., Gilden, D. H., \& Burgoon, M. P. (2006). The B cell response in multiple sclerosis. Neurological research, 28(3), 236-244.

29. Pandit, L., \& Murthy, J. (2011). Treatment of multiple sclerosis. Annals of Indian Academy of Neurology, 14(Suppl1), S65. 
30. Pröbstel, A.-K., Sanderson, N. S. R., \& Derfuss, T. (2015). B Cells and Autoantibodies in Multiple Sclerosis. International journal of molecular sciences, 16(7), 16576-16592. doi:10.3390/ijms160716576

31. Racke, M. K. (2001). Experimental autoimmune encephalomyelitis (EAE). Current protocols in neuroscience, 14(1), 9.7. 1-9.7. 11.

32. Reindl, M., Linington, C., Brehm, U., Egg, R., Dilitz, E., Deisenhammer, F., . . Berger, T. (1999). Antibodies against the myelin oligodendrocyte glycoprotein and the myelin basic protein in multiple sclerosis and other neurological diseases: a comparative study. Brain : a journal of neurology, 122(11), 2047-2056.

33. Rolfes, L., Pfeuffer, S., Ruck, T., Melzer, N., Pawlitzki, M., Heming, M., . . Meuth, S. G. (2019). Therapeutic Apheresis in Acute Relapsing Multiple Sclerosis: Current Evidence and Unmet Needs-A Systematic Review. Journal of clinical medicine, 8(10), 1623. doi:10.3390/jcm8101623

34. Rozenblum, G. T., Kaufman, T., \& Vitullo, A. D. (2014). Myelin basic protein and a multiple sclerosisrelated MBP-peptide bind to oligonucleotides. Molecular Therapy-Nucleic Acids, 3, e192.

35. Sadovnick, A. D., Dyment, D., \& Ebers, G. (1997). Genetic epidemiology of multiple sclerosis. Epidemiologic reviews, 19(1), 99.

36. Stork, L., Ellenberger, D., Beißbarth, T., Friede, T., Lucchinetti, C. F., Brück, W., \& Metz, I. (2018). Differences in the reponses to apheresis therapy of patients with 3 histopathologically classified immunopathological patterns of multiple sclerosis. JAMA neurology, 75(4), 428-435.

37. Stromnes, I. M., \& Goverman, J. M. (2006). Active induction of experimental allergic encephalomyelitis. Nature protocols, 1(4), 1810.

38. Stromnes, I. M., \& Goverman, J. M. (2006). Passive induction of experimental allergic encephalomyelitis. Nature protocols, 1(4), 1952-1960. doi:10.1038/nprot.2006.284

39. Suurmond, J., \& Diamond, B. (2015). Autoantibodies in systemic autoimmune diseases: specificity and pathogenicity. The Journal of clinical investigation, 125(6), 2194-2202.

40. Villar, L., Masjuan, J., González-Porqué, P., Plaza, J., Sadaba, M., Roldan, E., . . Alvarez-Cermeño, J. (2002). Intrathecal IgM synthesis in neurologic diseases: relationship with disability in MS. Neurology, 58(5), 824-826.

41. Voge, N. V., \& Alvarez, E. (2019). Monoclonal antibodies in multiple sclerosis: present and future. Biomedicines, 7(1), 20.

42. Warren, K. G., \& Catz, I. (1993). Increased synthetic peptide specificity of tissue-CSF bound anti-MBP in multiple sclerosis. Journal of neuroimmunology, 43(1-2), 87-96.

43. Weber, M. S., Hemmer, B., \& Cepok, S. (2011). The role of antibodies in multiple sclerosis. Biochimica et Biophysica Acta (BBA)-Molecular Basis of Disease, 1812(2), 239-245.

44. Wolswijk, G. (1998). Chronic stage multiple sclerosis lesions contain a relatively quiescent population of oligodendrocyte precursor cells. Journal of Neuroscience, 18(2), 601-609. 


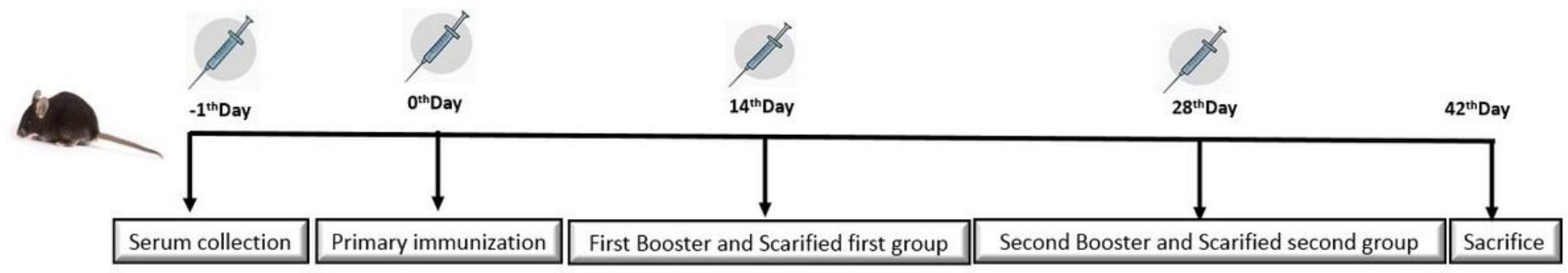

Figure 1

The experimental design for the immunization experiments.

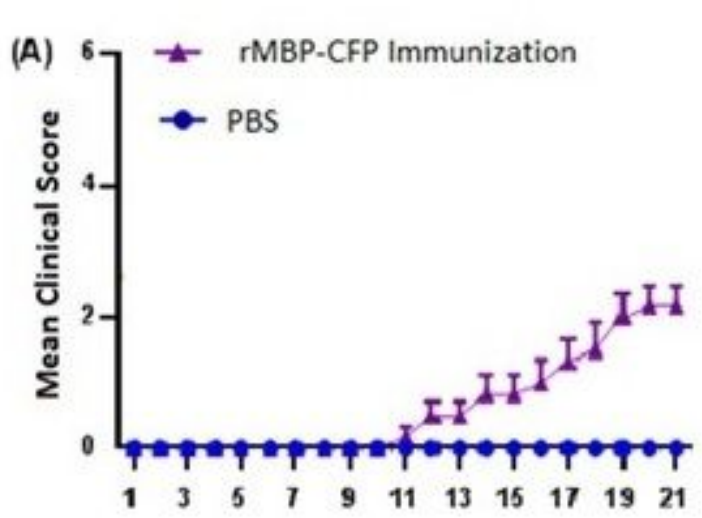

Days Post Immunization

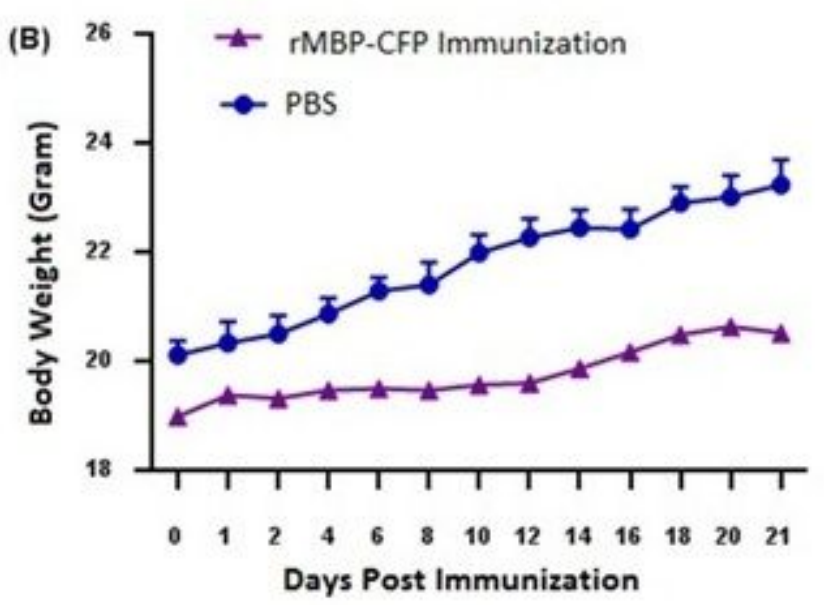

Days Post Immunization

\section{Figure 2}

Scores used for evaluation of symptoms of immunization with rMBP-CFP. (A) Mice were examined daily using a clinical EAE scoring system ranging from 0 (no signs) to 6 (complete paralysis), for 21 days. The mean clinical score of the control and test groups was: $0 \pm 0$ and $1.16 \pm 0.30(P<0.0001)$, respectively. (B) The average body weight of mice over the 3-week feeding study from control and test groups was: $3.43 \pm 0.22$ gram, and $2.10 \pm 0.22$ gram $(P<0.0001)$, respectively ( $n=4$ per group). 


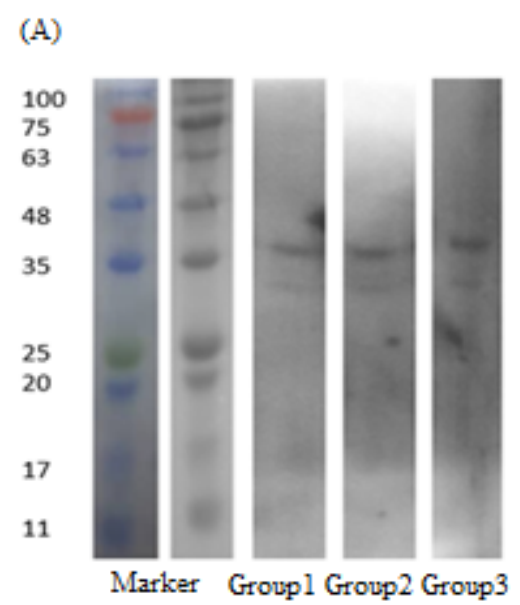

(B)

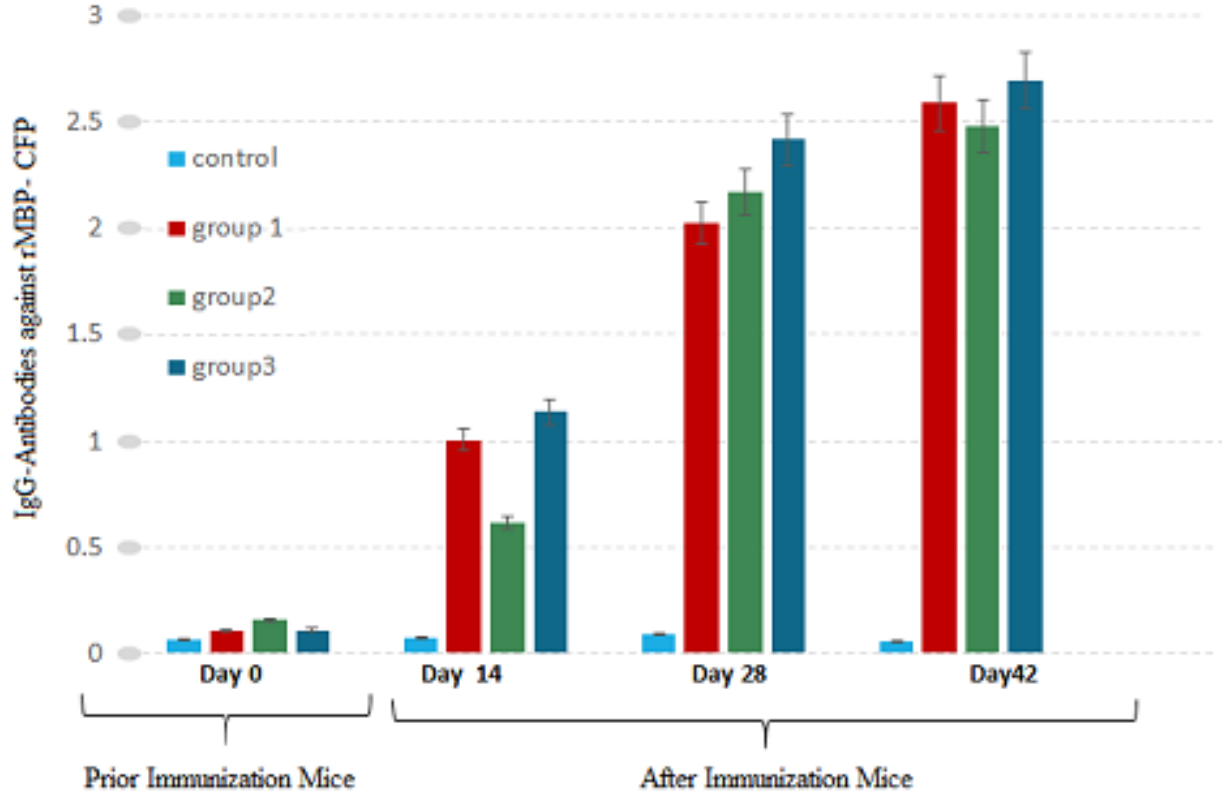

Figure 3

Humoral immune response to rMBP-CFP. (A) Western blot analysis of mice serum induced by rMBP- CFP peptide that was probed with a secondary anti-mice HRP (first column: day 0, second: 14 days, third: 28 days and fourth: 42 days after treatment). (B) ELISA analysis of antibodies against rMBP- CFP peptide in mice sera. Sera have been collected from each mouse prior to immunization and on day 0 and 14,28 , and 42 after immunization. (Each bar represents the mean \pm SD value of antibody responses from 4 mice from each group. $P<0.0001)$. 
(A)
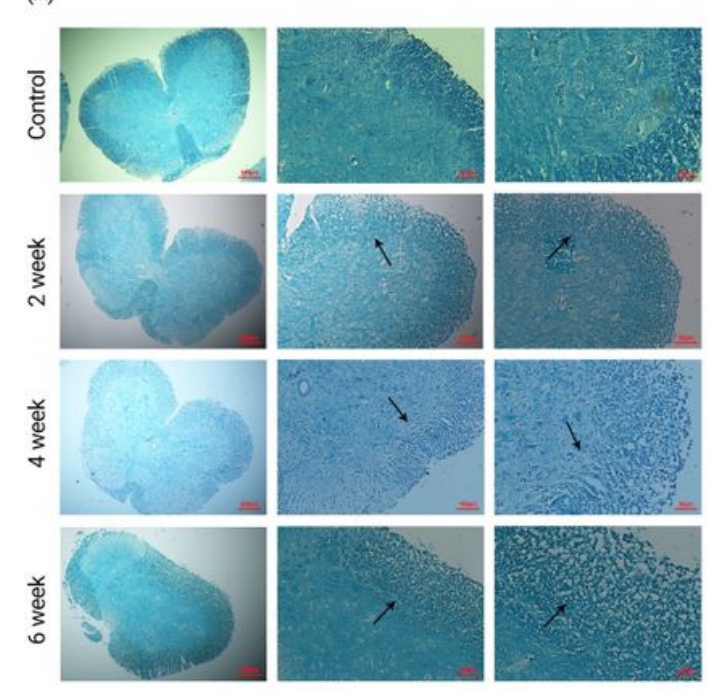

(B)
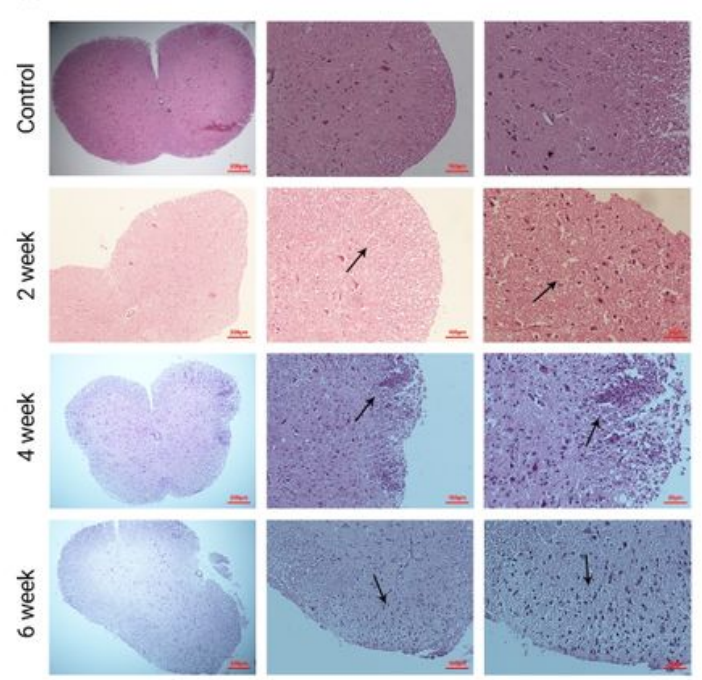

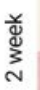

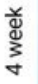

ఖँ

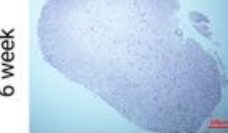

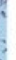

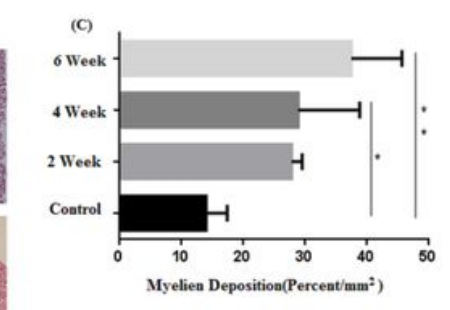$$
\text { . }
$$

\section{Figure 4}

Demyelination and inflammation in spinal cord. (A) Luxol fast blue staining in spinal cord sections of rMBP- CFP immunized animals revealed signs of demyelination indicated by white areas in the nerve. In control nerves, the myelin sheaths were uniformly stained (**, $p<0.0067)$. (B) H\&E stained spinal cord sections of rMBP- CFP immunized mice exhibited signs of inflammation compared to that in control group. (C)Tukey's Multiple Comparison Test showed 4 and 6 weeks after treat, the demyelinated areas in immunized mice were higher than control group $(p<0.05)$. The figures show one representative result from3 independent experiments. 
(A)
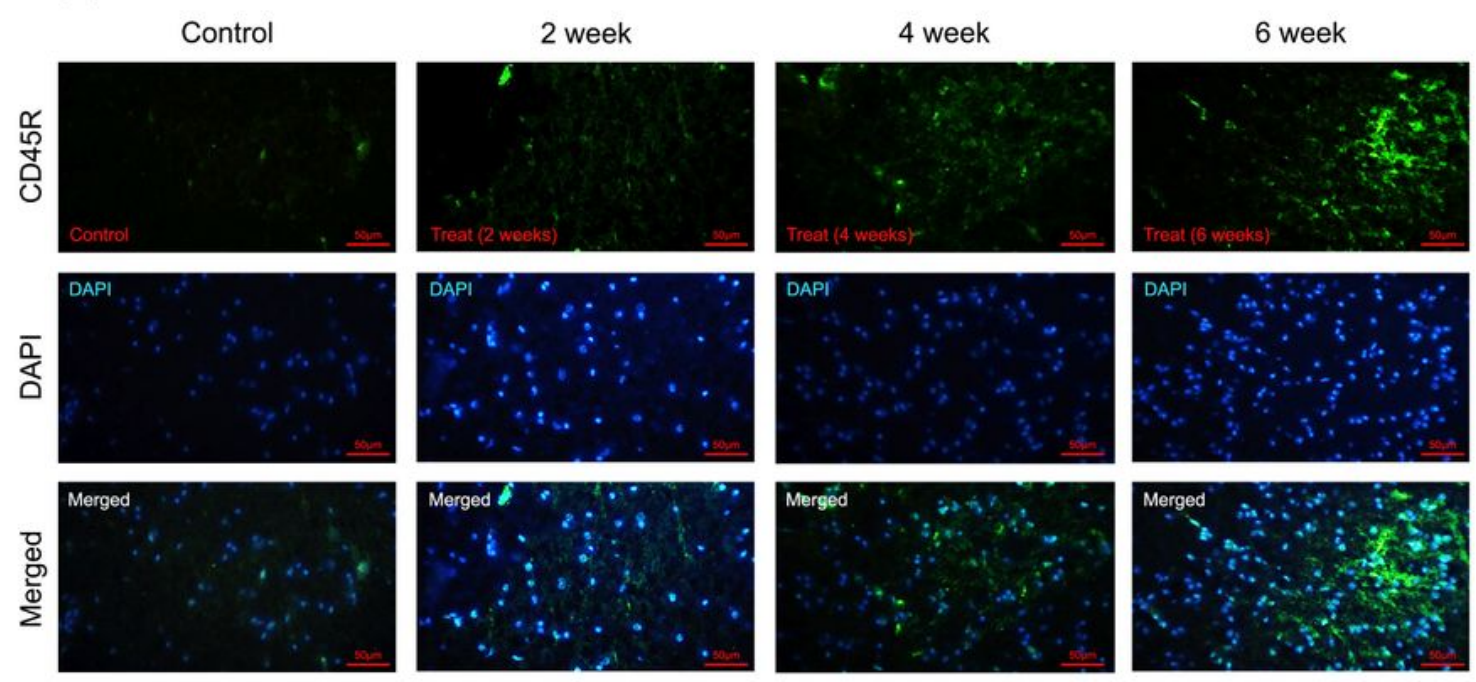

(B)
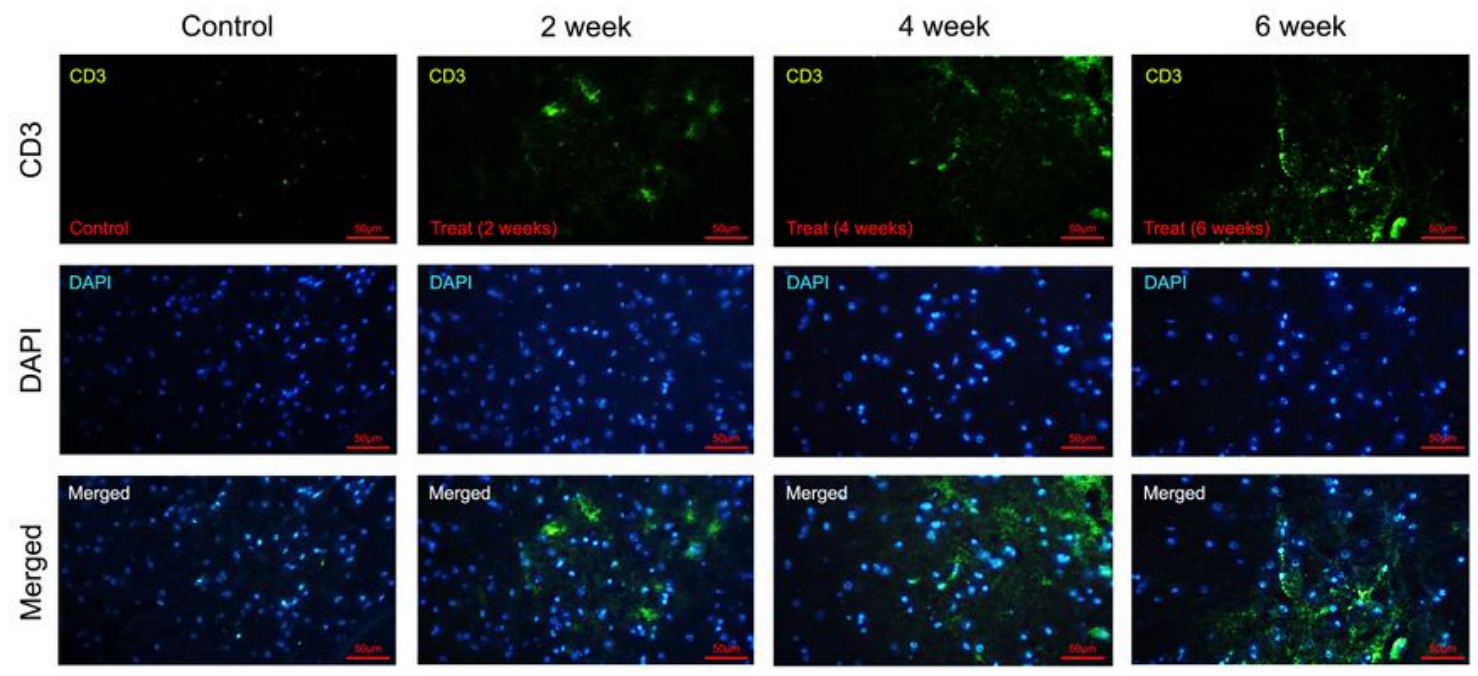

(C)
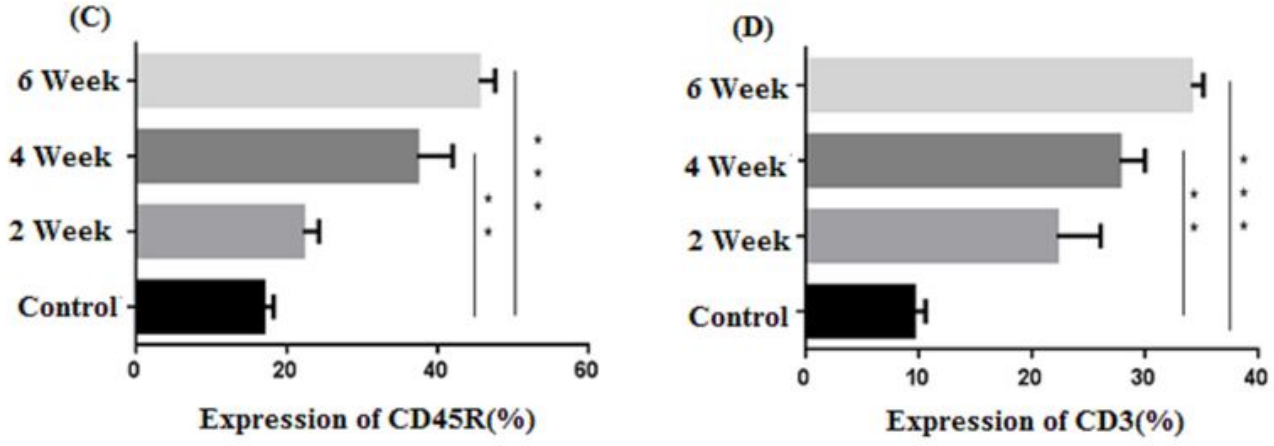

\section{Figure 5}

Autoreactive B cells and lymphocytes infiltrates in the lumbar spinal cord. (A) A significant increase in CD$45 \mathrm{R}+$ cells was detected in immunized mice with respect to control mice in the lumbar spinal cord (*ᄎ*, $p<0.009$ ). (B) An increase number of CD3+ cells was observed in the lumbar spinal cord of immunized mice compared to control (***, $p<0.006)$. (C) Tukey's Multiple Comparison Test showed the CD-45R+ cells was higher in the 6 weeks after treat than 2 weeks after treat $(p<0.05)$. (D) Tukey's Multiple Comparison 
Test elicited the higher number of CD3+ cells in the 6 weeks after treat than a 2-week after treat $(p<0.05)$. The data are representative of three separate experiments.
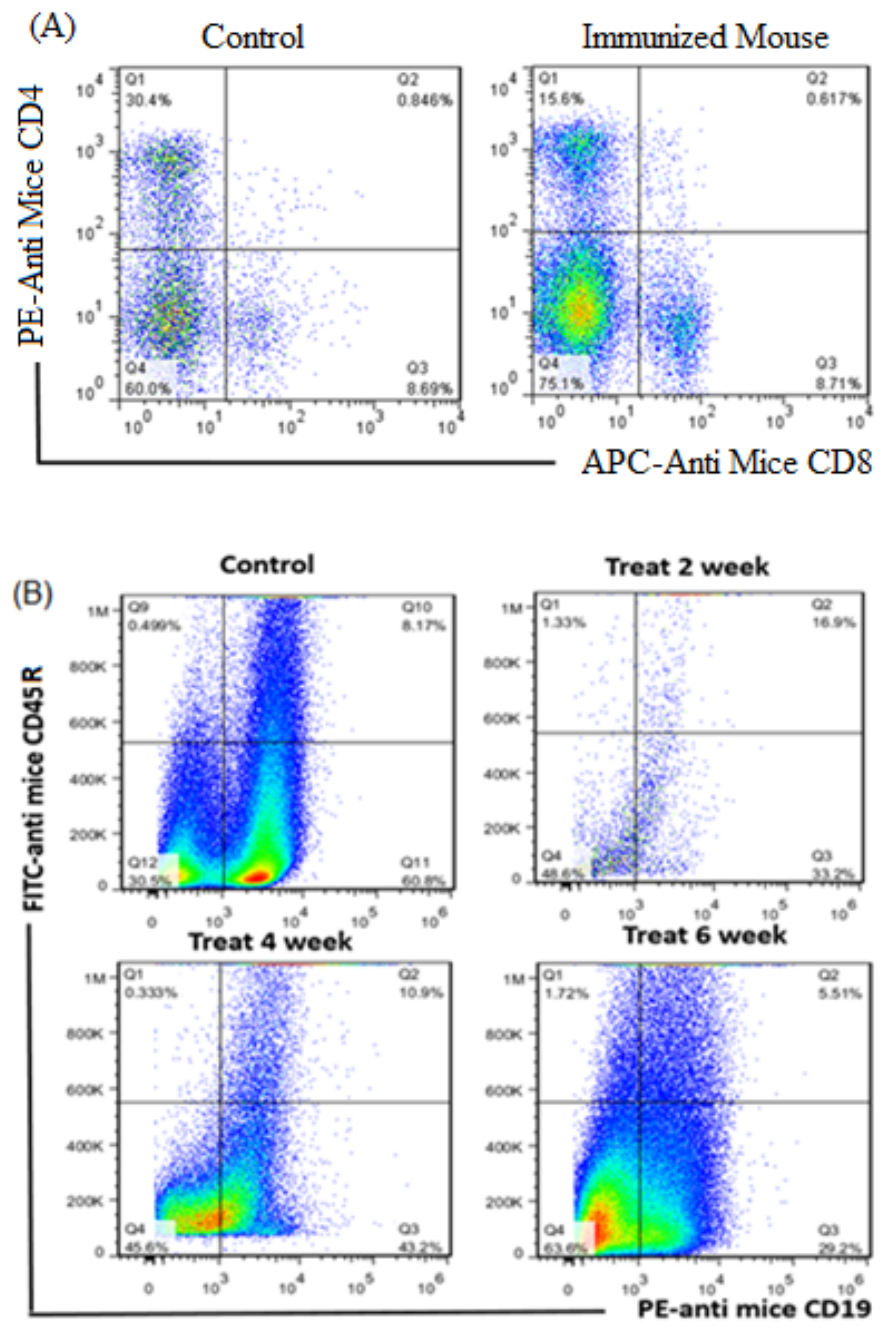

(C)
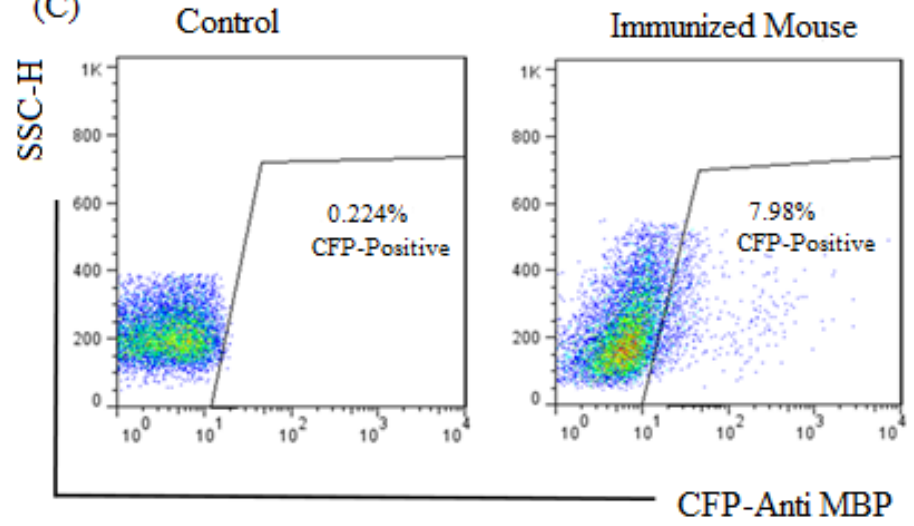

Figure 6

Cell mediate response in rMBP-CFP immunized mice. $(A)$ The ratio of CD4+ $(30.4 \%)$ and T CD $8+(8.69)$ lymphocytes. (B) The percentage of CD19+/CD45R+ fraction. (C) The percentage of CFP-positive cells as autoreactive B cell population. 